\title{
How efficient is patient discharge following lower limb arthroplasty?
}

Elizabeth Ashby ${ }^{1 *}$, Claire Matejowsky ${ }^{2}$, Michael G Mythen ${ }^{3}$, Fares S Haddad ${ }^{4}$ and Michael PW Grocott ${ }^{3,5,6}$

\begin{abstract}
Background: Appropriately timed patient discharge is essential for optimal patient care and efficient hospital functioning. The post-operative morbidity survey (POMS) is the only validated prospective method of measuring short-term post-operative morbidity. It has not previously been used as a bed utilisation tool.

Methods: We collected POMS data from 529 consecutive lower-limb arthroplasty patients over a 1-year period and recorded the number of patients remaining in the hospital without morbidity, together with alternative reasons for remaining in hospital. Data was collected on post-operative days (POD) 3, 5, 8 and 15.

Results: On POD 3, 45\% of hip arthroplasty patients and 52\% of knee arthroplasty patients remained in hospital with no identifiable morbidity. On POD 5, 53\% of hip arthroplasty patients and 47\% of knee arthroplasty patients remained in hospital with no identifiable morbidity. These figures declined by POD 8 and 15. The most common reason for inappropriate bed occupancy was ongoing physiotherapy and occupational therapy.

Conclusions: We believe POMS is able to identify patients remaining in hospital with no significant morbidity and has utility as a prospective bed utilisation tool. Addition of a mobility measure to POMS may improve its utility in detecting morbidity requiring hospitalisation, particularly following lower limb arthroplasty.
\end{abstract}

Keywords: Bed utilisation, Arthroplasty, Post-operative morbidity

\section{Background}

Appropriately timed discharge of patients following surgery is essential for optimal patient care and efficient hospital functioning. A patient discharged early is at risk of under-diagnosis of medical complications with consequent adverse outcome. A patient whose discharge is delayed is at risk of developing a hospital-associated complication (for example, hospital-acquired infection) and incurs an unnecessary cost to the health-care provider. Postoperative patients should be discharged at the earliest safe opportunity to reduce the rate of hospital-associated complications and the cost of each in-patient episode. Appropriate discharge timing should increase the throughput of patients and reduce waiting times.

Historically, hospitals in the UK have been paid according to contracts with no financial incentive to treat increased numbers of patients. This changed in 2000 when the National Health Service (NHS) Plan [1] announced

\footnotetext{
* Correspondence: elizabethashby@doctors.org.uk

${ }^{1}$ Catterall Unit, Royal National Orthopaedic Hospital, Brockley Hill, Stanmore, Middlesex HA7 4LP, UK

Full list of author information is available at the end of the article
}

that hospital incomes would be directly linked to activity. Payment by results [2] began in 2003 and now every healthcare provider is paid a sum (tariff) for each procedure undertaken. In the UK, many patients remain in hospitals with no medical indication [3]. One study showed that $31 \%$ of post-operative patients remained in hospitals inappropriately [4]. Payment by results aims to reduce this figure by rewarding efficiency and encouraging increased activity.

In order to improve efficiency, hospitals must first recognise inappropriate bed occupancy. The post-operative morbidity survey (POMS) [5] is the only validated prospective method of measuring short-term post-operative morbidity in the literature. POMS was designed to identify morbidity of a type and severity that could delay hospital discharge. The survey focuses on indicators of organ system dysfunction (for example, inability to tolerate enteral diet) rather than traditional diagnostic categories (for example, deep vein thrombosis). POMS assesses nine domains of morbidity (Table 1). Data is obtained from observation charts, medication charts, patient 
Table 1 Criteria for a positive POMS score

\begin{tabular}{|c|c|}
\hline Variable & Criteria for positive result \\
\hline Pulmonary & $\begin{array}{l}\text { Requires supplementary oxygen or ventilatory } \\
\text { support }\end{array}$ \\
\hline Infection & $\begin{array}{l}\text { Currently on antibiotics or temperature }>38^{\circ} \mathrm{C} \text { in } \\
\text { the last } 24 \text { hours }\end{array}$ \\
\hline Renal & $\begin{array}{l}\text { Oliguria (<500 ml/day), elevated creatinine } \\
\text { (>30\% pre-op level), catheter in situ (for } \\
\text { non-surgical reason) }\end{array}$ \\
\hline Gastrointestinal & Unable to tolerate enteral diet for any reason \\
\hline Cardiovascular & $\begin{array}{l}\text { Diagnostic tests or treatment within the last } \\
24 \text { h for: myocardial infarction, hypotension } \\
\text { (requiring pharmacological therapy or fluids > } \\
200 \mathrm{ml} / \mathrm{h} \text { ), atrial/ventricular arrhythmia or } \\
\text { cardiogenic pulmonary oedema }\end{array}$ \\
\hline $\begin{array}{l}\text { Central nervous } \\
\text { system }\end{array}$ & $\begin{array}{l}\text { Presence of new focal deficit, coma, confusion or } \\
\text { delirium }\end{array}$ \\
\hline $\begin{array}{l}\text { Wound } \\
\text { complications }\end{array}$ & $\begin{array}{l}\text { Wound dehiscence requiring surgical exploration or } \\
\text { drainage of pus from operative wound with or } \\
\text { without isolation of organisms }\end{array}$ \\
\hline Haematological & $\begin{array}{l}\text { Requirement of blood transfusion, platelets, fresh } \\
\text { frozen plasma, or cryoprecipitate within the last } 24 \mathrm{~h}\end{array}$ \\
\hline Pain & $\begin{array}{l}\text { Wound pain requiring parenteral opioids or regional } \\
\text { anaesthesia }\end{array}$ \\
\hline
\end{tabular}

notes, routine blood test results and direct patient questioning and observation. No additional investigations are required. The data collection process is simple to allow routine screening of large numbers of patients. POMS has been shown to be reliable, valid and acceptable to patients [6]. POMS has been used in outcomes research [7] and in effectiveness research [8].

In the US, over $98 \%$ of post-operative in-patients had morbidity defined by POMS [5]. This implies that patients with a POMS score of 0 are fit for discharge.

Therefore, as well as providing useful clinical research and audit data, POMS may have utility for assessing and improving hospital bed utilisation.

The aim of this study is to identify inappropriate bed occupancy following lower limb arthroplasty using POMS. We report the reasons for delayed discharge and suggest ways to improve bed utilisation.

\section{Methods}

Ethical approval was gained from the Joint UCLH/UCL Committee on the Ethics of Human Research (ref. number 01/0116) prior to commencement of the study. The requirement for consent was waived as collection of POMS has become a routine part of service evaluation within our institution. All patients aged 18 or over undergoing elective lower limb arthroplasty at University College Hospitals NHS Trust over a 12-month period were eligible for inclusion into this prospective cohort study. There were no exclusion criteria ensuring a consecutive sample was taken. Elective lower limb arthroplasty procedures included unicompartmental knee replacement (UKR), total knee replacement (TKR), revision total knee replacement, hip resurfacing (HR), total hip replacement (THR) and revision total hip replacement.

Data was collected by one of two study nurses. The age, sex, American Society of Anesthesiologists (ASA) physical status score and length of in-patient stay for each patient were recorded. POMS data were collected on postoperative days (POD) 3, 5, 8 and 15 if the patient remained in hospital. Presence of morbidity was defined as occurring in any patient meeting POMS criteria in one or more domains of the survey on the day of data collection. For patients remaining in hospital without morbidity on POD 8 and POD 15, the reason was recorded. The use of mobility aids on these days was also noted.

The number and percentage of patients with no identifiable morbidity according to POMS was calculated for POD 3, 5, 8 and 15. The number of days each patient remained in hospital with no morbidity was calculated by distracting the day on which the patient first had a POMS score of 0 from his or her total length of stay. An overall estimated cost saving was calculated by multiplying this figure by the average cost for one orthopaedic in-patient night.

The number of patients developing postoperative morbidity after a period free of morbidity was recorded. The number of readmissions to the same hospital in the first year following discharge was also recorded.

\section{Results}

Data collection was completed on 529 patients. Patient characteristics of the study population are shown in Table 2. The mean age of all study patients was 68.9 years, the median ASA physical status score was 2 and $62 \%$ of patients were female. The median length of stay was 7 days, and the overall in-patient mortality rate was $0.4 \%$.

\section{A) Hip arthroplasty patients}

The location of hip arthroplasty patients on POD 3, 5, 8 and 15 is shown in Table 3. Many patients undergoing HR remained in the hospital with no identified morbidity on POD $3(75 \%), 5(78 \%)$ and $8(16 \%)$. All HR patients had been discharged by POD 15 .

Many THR patients remained in hospital with no identifiable morbidity on POD 3 (46\%), 5 (54\%), 8(34\%) and 15 (7\%). Patients undergoing revision THR patients remained in the hospital with no identifiable morbidity on POD 3 (11\%), 5 (29\%), 8 (31\%) and 15 (3\%). Discharge status and prevalence of morbidity for all hip arthroplasty patients combined are presented in Figure 1.

\section{B) Knee arthroplasty patients}

The location of knee arthroplasty patients on POD 3, 5, 8 and 15 is shown in Table 4. Many patients undergoing 
Table 2 Demographics of the study population

\begin{tabular}{|c|c|c|c|c|c|c|c|c|c|}
\hline & \multirow{2}{*}{$\begin{array}{l}\text { Number } \\
\text { performed }\end{array}$} & \multirow{2}{*}{$\begin{array}{l}\text { Mortality } \\
\text { rate (\%) }\end{array}$} & \multicolumn{2}{|c|}{ Age (years) } & \multicolumn{2}{|c|}{ ASA physical status score } & \multirow[t]{2}{*}{$\%$ Male } & \multicolumn{2}{|c|}{ Length of stay (days) } \\
\hline & & & Mean & Range & Median & Range & & Median & Range \\
\hline UKR & 66 & 1 & 66.1 & 45 to 87 & 2 & 1 to 3 & 45 & 5 & 2 to 52 \\
\hline TKR & 226 & 0 & 70.3 & 23 to 90 & 2 & 1 to 3 & 36 & 6 & 3 to 37 \\
\hline RTKR & 8 & 0 & 71.6 & 46 to 88 & 2 & 1 to 3 & 25 & 13 & 3 to 102 \\
\hline$H R$ & 32 & 0 & 51.6 & 22 to 70 & 1 & 1 to 3 & 50 & 6 & 4 to 13 \\
\hline THR & 162 & 0 & 70.7 & 21 to 89 & 2 & 1 to 3 & 36 & 8 & 3 to 51 \\
\hline RTHR & 35 & 3 & 72.2 & 26 to 88 & 2 & 1 to 3 & 36 & 14 & 6 to 93 \\
\hline Total & 529 & 0.4 & 68.9 & 21 to 90 & 2 & 1 to 3 & 38 & 7 & 2 to 102 \\
\hline
\end{tabular}

UKR remained in the hospital with no identified morbidity on POD 3 (63\%), 5 (39\%) and 8 (6\%). All UKR patients had been discharged by POD 15 .

Many TKR patients remained in the hospital with no identifiable morbidity on POD 3 (50\%), 5 (50\%), 8 (19\%) and 15 (4\%). Revision TKR patients remained in the hospital with no identifiable morbidity on POD 3 (52\%), 5 (47\%), 8 (17\%) and 15 (3\%). Discharge status and prevalence of morbidity for all knee arthroplasty patients combined are presented in Figure 2.

\section{C) Overall inappropriate bed occupancy days}

Table 5 shows the average number of days that postoperative patients remained in the hospital with no identifiable morbidity. HR patients stayed an average of 2.36 days with no morbidity, THR patients 4.19 days and revision THR patients 10.37 days. UKR patients stayed an average of 1.76 days with no identifiable morbidity, TKR patients 2.73 days and revision TKR patients 14.38 days.

\section{D) Cost of inappropriate bed occupancy days}

529 patients were included in this study. These patients remained in the hospital for a total of 1,965 days with no morbidity as defined by POMS. A surgical in-patient bed costs up to $£ 400$ per night [9]. If these patients had been discharged when their POMS score was 0 , a saving of up to $£ 786,000$ could have been made.

\section{E) Reasons for patients with no morbidity remaining in hospital}

Of the 529 patients participating in this study, 120 remained in hospital with no morbidity defined by POMS on POD 8, and 20 patients remained with no identifiable morbidity on POD 15 . The reasons for nondischarge are shown in Figure 3. The most common reason is continuing physiotherapy and occupational therapy input. Other reasons include waiting for home equipment, waiting for a rehabilitation bed, waiting for a social services package of care and patients feeling unwell with negative investigations.

Of the patients remaining in hospital with no morbidity identified by POMS, $24 \%$ were mobilising with a zimmer frame, 55\% were mobilising with two crutches, $14 \%$ with a single crutch and $7 \%$ were mobilising unaided. This study did not record how far patients could mobilise or whether they could climb stairs.

Table 3 Location of patients following hip arthroplasty

\begin{tabular}{|c|c|c|c|c|c|c|}
\hline & & & POD 3 & POD 5 & POD 8 & POD 15 \\
\hline \multirow[t]{12}{*}{ Procedure } & $\mathrm{HR}$ & Patients discharged & $0 / 32(0 \%)$ & $2 / 32(6 \%)$ & 27/32 (84\%) & $32 / 32(100 \%)$ \\
\hline & & Inpatients POMS >0 & $8 / 32(25 \%)$ & $5 / 32(16 \%)$ & $0 / 32(0 \%)$ & $0 / 32(0 \%)$ \\
\hline & & Inpatients $\mathrm{POMS}=0$ & 24/32 (75\%) & 25/32 (78\%) & $5 / 32(16 \%)$ & 0/32 (0\%) \\
\hline & THR & Patients discharged & 0/162 (0\%) & 13/162 (8\%) & 78/162 (48\%) & 138/162 (85\%) \\
\hline & & Inpatients POMS >0 & $87 / 162(54 \%)$ & $62 / 162(38 \%)$ & 29/162 (18\%) & 13/162 (8\%) \\
\hline & & Inpatients $\mathrm{POMS}=0$ & 75/162 (46\%) & $87 / 162$ (54\%) & $55 / 162(34 \%)$ & 11/162 (7\%) \\
\hline & Revision THR & Patients discharged & 0/35 (0\%) & 0/35 (0\%) & 3/35 (9\%) & 20/35 (57\%) \\
\hline & & Inpatients POMS >0 & 31/35 (89\%) & 25/35 (71\%) & $21 / 35(60 \%)$ & $14 / 35(40 \%)$ \\
\hline & & Inpatients $\mathrm{POMS}=0$ & $4 / 35(11 \%)$ & 10/35 (29\%) & 11/35 (31\%) & 1/35 (3\%) \\
\hline & TOTAL & Patients discharged & 0/230 (0\%) & 16/230 (7\%) & 109/230 (47\%) & $191 / 230(83 \%)$ \\
\hline & & Inpatients POMS >0 & $127 / 230(55 \%)$ & $92 / 230(40 \%)$ & $50 / 230(22 \%)$ & $27 / 230(12 \%)$ \\
\hline & & Inpatients $\mathrm{POMS}=0$ & $103 / 230(45 \%)$ & $122 / 230(53 \%)$ & $71 / 230$ (31\%) & $12 / 230(5 \%)$ \\
\hline
\end{tabular}




\section{All hip arthroplasty patients}

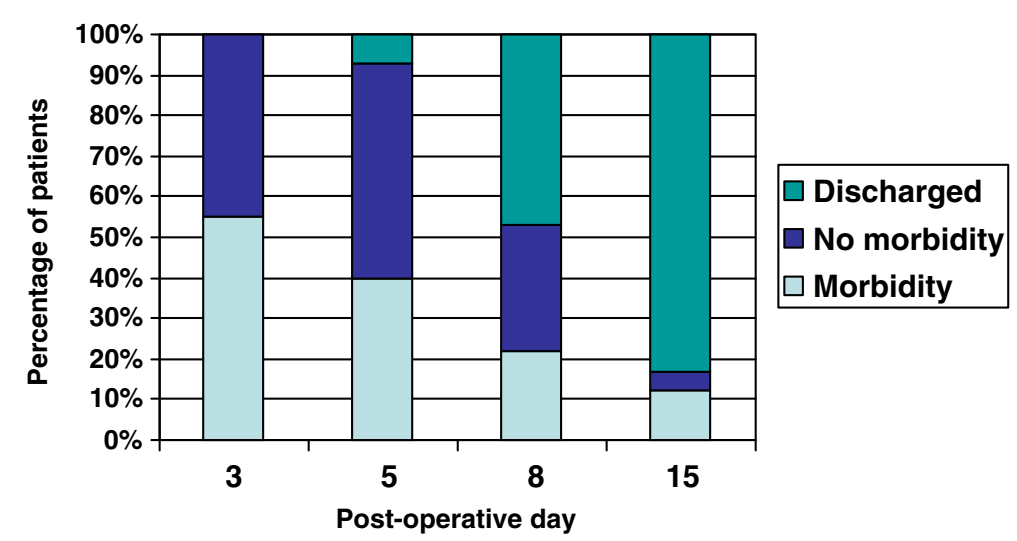

Figure 1 Discharge status and prevalence of morbidity following all types of hip arthroplasty.

F) New morbidity and readmission

Thirty-eight out of 529 patients developed morbidity as an in-patient following a period without morbidity. Five of these patients underwent a second surgical procedure and developed morbidity in the second post-operative period. Thirty patients (6.2\%) developed morbidity following a period without morbidity. Twenty-five of these patients developed wound morbidity: 24 patients had no morbidity on POD 3 but developed wound morbidity by POD 5, and 1 revision arthroplasty patient had no morbidity on POD 5 but developed wound morbidity by POD 8. Six patients developed cardiovascular morbidity after a period with no morbidity: 5 patients were prescribed anticoagulation (2 for pulmonary embolus and 3 for deep vein thrombosis) and 1 patient had a myocardial infarction. One patient developed neurological morbidity (a cerebro-vascular accident) after a period without morbidity, and 1 patient developed infectious morbidity (an infected peripheral cannula site) after a period without morbidity.
No patient in this study was readmitted to the same hospital in the first year following discharge for any reason relating to their surgery.

\section{Discussion}

This study identifies many patients remaining in hospital with no identifiable morbidity following lower-limb arthroplasty in a UK teaching hospital. The rate of inappropriate bed occupancy varies with time after surgery and type of arthroplasty.

The most common reason for patients remaining in hospital with no identifiable morbidity was ongoing physiotherapy and occupational therapy. This suggests that improving pre- and post-operative planning could improve appropriate bed occupancy. Patients could be taught post-operative physiotherapy exercises in group classes prior to surgery. Occupational therapists could assess each patient's home environment and ensure necessary modifications are made. In the post-operative

Table 4 Location of patients following knee arthroplasty

\begin{tabular}{|c|c|c|c|c|c|c|}
\hline & & & Day 3 post-op & Day 5 post-op & Day 8 post-op & Day 15 post-op \\
\hline \multirow[t]{12}{*}{ Procedure } & \multirow[t]{3}{*}{ UKR } & Patients discharged & 7/66 (11\%) & $33 / 66(50 \%)$ & $59 / 66(89 \%)$ & 65/66 (98\%) \\
\hline & & Inpatients POMS >0 & $17 / 66(26 \%)$ & $7 / 66(11 \%)$ & $3 / 66(5 \%)$ & $1 / 66(2 \%)$ \\
\hline & & Inpatients $\mathrm{POMS}=0$ & 42/66 (63\%) & 26/66 (39\%) & $4 / 66(6 \%)$ & 0/66 (0\%) \\
\hline & \multirow[t]{3}{*}{ TKR } & Patients discharged & $0 / 226(0 \%)$ & $22 / 226(10 \%)$ & $145 / 226(64 \%)$ & $211 / 226(93 \%)$ \\
\hline & & Inpatients POMS >0 & $114 / 226(50 \%)$ & $90 / 226(40 \%)$ & $38 / 226(17 \%)$ & $7 / 22(3 \%)$ \\
\hline & & Inpatients $\mathrm{POMS}=0$ & $112 / 226(50 \%)$ & $114 / 226(50 \%)$ & $43 / 226(19 \%)$ & $8 / 226(4 \%)$ \\
\hline & \multirow[t]{3}{*}{ Revision TKR } & Patients discharged & 0/8 (0\%) & $1 / 8(13 \%)$ & $1 / 8(13 \%)$ & $6 / 8(75 \%)$ \\
\hline & & Inpatients POMS >0 & $6 / 8(75 \%)$ & $4 / 8(50 \%)$ & $5 / 8(62 \%)$ & $1 / 8(12.5 \%)$ \\
\hline & & Inpatients $\mathrm{POMS}=0$ & $2 / 8(25 \%)$ & $3 / 8(37 \%)$ & $2 / 8(25 \%)$ & $1 / 8(12.5 \%)$ \\
\hline & \multirow[t]{3}{*}{ TOTAL } & Patients discharged & $7 / 300(2 \%)$ & $56 / 300(19 \%)$ & $205 / 300(68 \%)$ & $282 / 300(94 \%)$ \\
\hline & & Inpatients POMS >0 & $137 / 300(46 \%)$ & $101 / 300$ (34\%) & $46 / 300(15 \%)$ & $9 / 300(3 \%)$ \\
\hline & & Inpatients $\mathrm{POMS}=0$ & $156 / 300(52 \%)$ & $143 / 300$ (47\%) & 49/300 (17\%) & $9 / 300(3 \%)$ \\
\hline
\end{tabular}




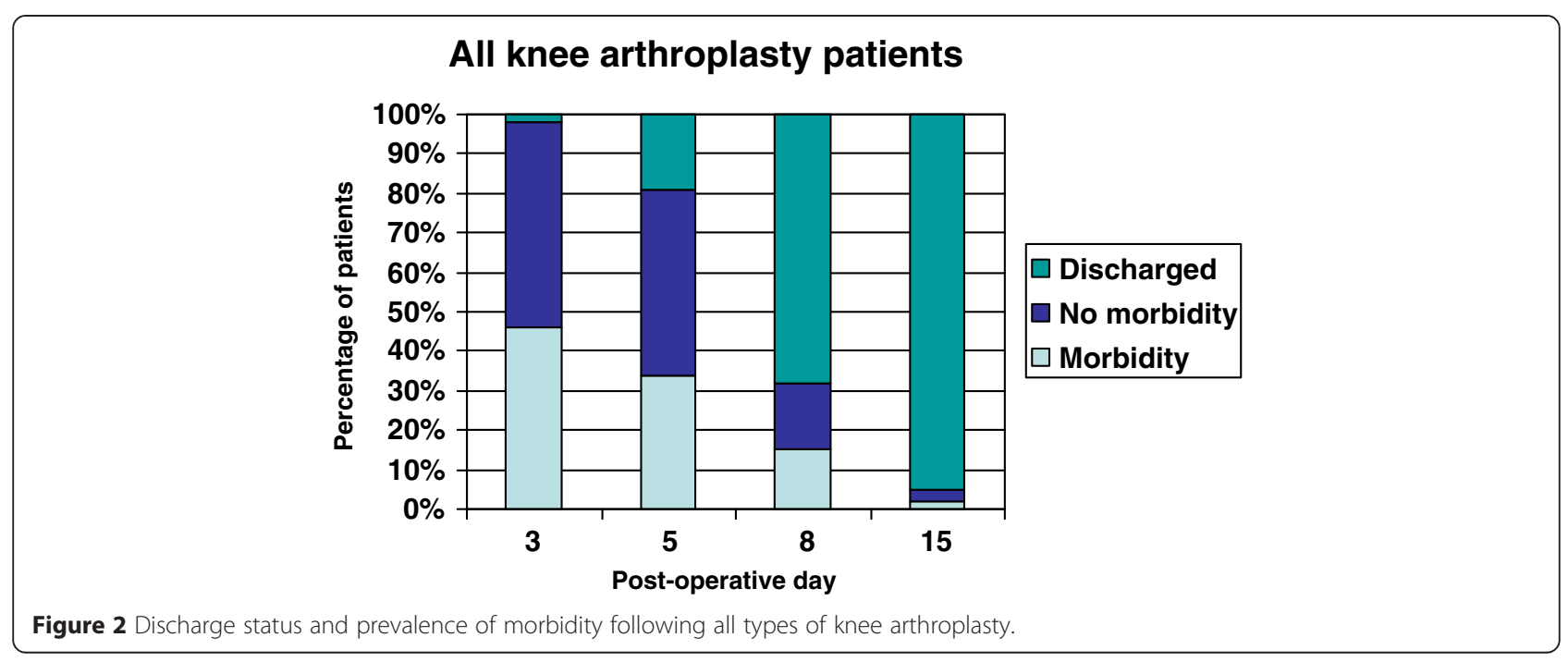

period 'fast-track' pathways could be used to ensure maximum therapy input at the earliest possible opportunity. Some physiotherapy and occupational therapy could be provided post-operatively in a rehabilitation unit or at the patient's home rather than in hospital. This would require safety and cost evaluation prior to implementation.

Three of the top five reasons for patients remaining in hospital with no identifiable morbidity relate to 'social' issues (awaiting home equipment, awaiting a rehabilitation bed, awaiting a package of care from social services). Pre-operative clinics could identify and address these problems prior to admission. Such clinics could also be used to manage patient expectation so they are aware of the difficulties they may encounter in the postoperative period and the expected timing of discharge.

This study has several strengths. A large consecutive dataset was collected prospectively using a validated methodology for measuring post-operative morbidity. This is the first published study to prospectively evaluate

Table 5 Number of inappropriate inpatient days classified by type of arthroplasty

\begin{tabular}{llll}
\hline & $\begin{array}{l}\text { Total number } \\
\text { of patients }\end{array}$ & $\begin{array}{l}\text { Total number } \\
\text { of inappropriate } \\
\text { in-patient days }\end{array}$ & $\begin{array}{l}\text { Average number } \\
\text { of inappropriate } \\
\text { in-patient days } \\
\text { per patient }\end{array}$ \\
\hline HR & 33 & 78 & 2.36 \\
THR & 162 & 678 & 4.19 \\
Revision THR & 35 & 363 & 10.37 \\
UKR & 63 & 111 & 1.76 \\
TKR & 227 & 620 & 2.73 \\
Revision TKR & 8 & 115 & 14.38 \\
Total & 528 & 1965 & 3.72 \\
\hline
\end{tabular}

the appropriateness of discharge following lower limb joint replacement surgery.

The weaknesses of this study are that it was conducted in a single centre, POMS is not validated as a bed utilisation tool, there was not daily recording of data so the calculation of excess days are an approximation, and patient mobility was not fully assessed. Data was collected regarding mobility aids, but the distance each patient could mobilise was not recorded.

This is the first time that POMS has been used as a bed utilisation tool. It has not been validated for this purpose but has previously been used to identify patients in hospitals without morbidity [5,6]. In the US, over $98 \%$ of in-patients had morbidity defined by POMS [5] suggesting that patients with POMS score of 0 were rapidly discharged. In a previous UK study, $63 \%$ of orthopaedic patients remained in hospital with no morbidity on POD 3 and $42 \%$ on POD 5 suggesting that discharge efficiency was lower in the UK institution. In the US, many postoperative arthroplasty patients are discharged to a rehabilitation unit. This practice is far less common in the UK and may partially account for the discrepancy in discharge efficiency.

Use of POMS as a bed utilisation tool relies on the assumption that it captures all reasons for remaining in hospital. In this study, the main reason for remaining in hospital with no identifiable morbidity was 'ongoing physiotherapy and occupational therapy input'. A specific concern in this patient group, who are often elderly and frail, is that they may not have adequate mobility to be discharged safely. Including a specific domain for mobility may improve the sensitivity of POMS for morbidity requiring hospitalisation following orthopaedic surgery. The domain should assess the ability to walk short distances, ability to climb a flight of stairs and a balance and falls assessment. Whilst this domain could be 


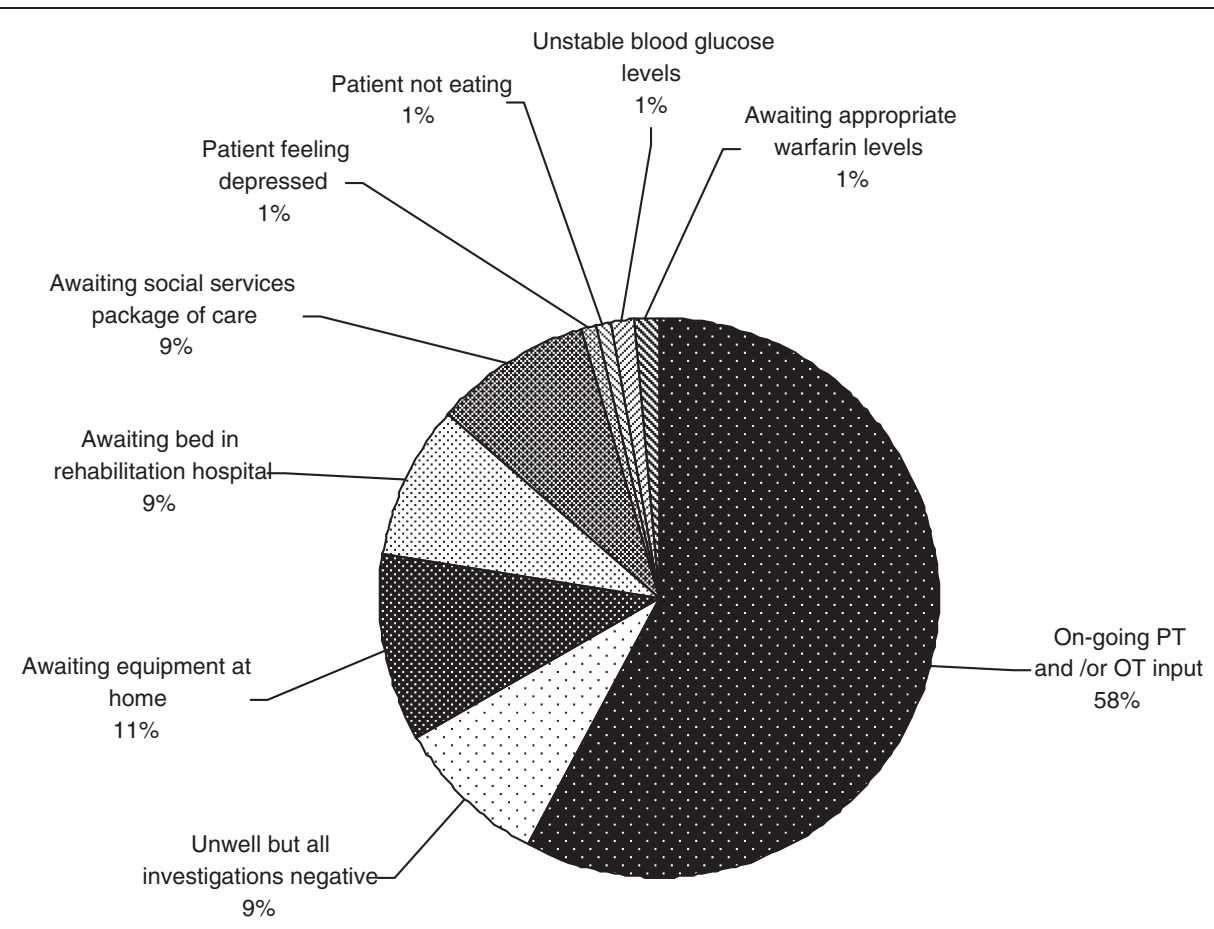

Figure 3 Reasons lower-limb arthroplasty patients with no morbidity remained in the hospital on post-operative days 8 and 15.

especially relevant for orthopaedic patients, this requires further investigation.

Use of POMS as a 'fitness for discharge' tool rests on the assumption that patients do not develop new morbidity after they have become free from morbidity, either in hospital or following discharge. No patients were readmitted to the study hospital in the first post-operative year for complications linked to surgery. However, 33 patients (6.2\%) developed 'new' morbidity following a period without morbidity whilst in hospital. This highlights a limitation of prospectively using POMS as a 'fitness for discharge' tool. 25 of 33 (76\%) of these patients developed wound morbidity after a period with no morbidity. To overcome this potential problem, primary arthroplasty patients should have regular wound reviews until POD 5 and revision arthroplasty patients until POD 8 . This could be performed in an outpatient or primary care setting by a doctor or nurse. Extra capacity would be needed to ensure no negative impact on existing services. This would add cost to each patient episode but would be more cost effective than patients remaining in hospital. Patients should be aware of the risk of deep vein thrombosis, pulmonary embolus, myocardial infarction and cerebro-vascular accident following discharge and receive clear written instructions regarding symptoms and management. As long as these precautionary measures are in place, POMS has potential as a bed utilisation tool.

The most commonly used tool to assess appropriate bed utilisation in the literature is the Appropriateness
Evaluation Protocol (AEP) [10]. AEP is a retrospective tool that relies on data from the inpatient record. It has been shown to be valid and reliable in some studies [10] but not in others [11]. POMS is a prospective tool that could be used in real time to assist with appropriate patient discharge. AEP is a retrospective tool that can only be used to evaluate past events. Data for POMS is collected directly from contemporary data sources whilst the patient is in hospital; AEP relies solely on past patient records and is therefore dependent on completeness and accuracy of record keeping for reliable functioning.

AEP has been used in several European countries to examine bed utilisation. In Portugal, $50 \%$ of inpatient days were deemed inappropriate [12], in Italy $37.3 \%$ [3], in Germany 28\% [13], in Switzerland 8\% to 15\% [14] and in France 7\% [15]. This study indicates bed utilisation in the UK is comparable to that seen in Portugal and Italy but such a direct comparison may have limited validity since different bed utilisation tools have been used.

The finding that many fewer patients remain in the hospital with no morbidity (as defined by POMS) in the US when compared with the UK suggests that bed utilisation in the US is superior to that seen in the UK. The implementation of 'payment by results' in the UK aims to improve appropriate bed occupancy to optimise patient care and improve efficiency. If the patients in this study had been discharged when they first had no morbidity defined by POMS, a saving of over $£ 750,000$ 
could have been made in 1 year (based on a cost of $£ 400$ per bed-day). This money could be reinvested in rehabilitation services with a strong emphasis on therapies rather than being spent on unnecessary inpatient medical care.

\section{Conclusions}

We believe that POMS is able to identify patients remaining in hospital without clinically significant morbidity and may be used prospectively as a bed utilisation tool. To use the survey for this purpose, it may be useful to add an additional measure to assess mobility.

\section{Abbreviations}

AEP: appropriateness evaluation protocol; ASA: American Society of Anesthesiologists; HR: hip resurfacing; POD: post-operative day; POMS: post-operative morbidity survey; THR: total hip replacement; TKR: total knee replacement; UK: United Kingdom; UKR: unicondylar knee replacement; US: United States (of America).

\section{Competing interests}

The authors declare that they have no competing interests.

\section{Authors' contributions}

EA participated in the design of the study, analysed the data and wrote the manuscript. CM collected data and helped with data analysis. MGM and FSH participated in the design and coordination of the study as well as interpretation of the data. MPWG participated in the study design, data analysis and helped to write the manuscript. All authors read and approved the final manuscript.

\section{Authors' information}

EA is an orthopaedic registrar at the Royal National Orthopaedic Hospital, Middlesex. CM is affiliated to the Surgical Outcomes Research Centre, University College London Hospitals. MGM is a medical professor of anaesthesia at the UCL Institute of Child Health; Surgical Outcomes Research Centre, Joint UCH/UCL Comprehensive Biomedical Research Centre. FSH is a professor of orthopaedic surgery at the University College London Hospital. MPWG is a professor of Anaesthesia and Critical Care Medicine, Integrative Physiology and Critical IIIness Group, Clinical and Experimental Sciences at the University of Southampton; University Hospital Southampton/University of Southampton NIHR Respiratory Biomedical Research Unit; senior lecturer at the UCL Institute of Child Health and at the Surgical Outcomes Research Centre, Joint UCH/UCL Comprehensive Biomedical Research Centre.

\section{Acknowledgements}

We would like to acknowledge Maj Mutch for collecting much of the data and Ramani Moonesinghe for managing the data.

\section{Funding}

Funding was received from the Special Trustees of the Middlesex Hospital and University College Hospital/UCL NIHR Biomedical Research Centre.

\footnotetext{
Author details

${ }^{1}$ Catterall Unit, Royal National Orthopaedic Hospital, Brockley Hill, Stanmore, Middlesex HA7 4LP, UK. ${ }^{2}$ Surgical Outcomes Research Centre, University College London Hospital, 235 Euston Road, Fitzrovia, London NW1 2BU, UK. ${ }^{3}$ UCL Institute of Child Health, Surgical Outcomes Research Centre, Joint UCLH/UCL Comprehensive Biomedical Research Centre, Gower Street, London WC1E 6BT, UK. ${ }^{4}$ University College London Hospital, 235 Euston Road, Fitzrovia, London NW1 2BU, UK. ${ }^{5}$ Integrative Physiology and Critical Illness Group, Clinical and Experimental Sciences, University of Southampton, University Road, Southampton SO17 1BJ, UK. ${ }^{6} \mathrm{NIHR}$ Respiratory Biomedical Research Unit, University Hospital Southampton/University of Southampton, University Road, Southampton SO17 1BJ, UK.
}

Received: 21 November 2014 Accepted: 30 March 2015

Published online: 30 April 2015

\section{References}

1. UK Department of Health. The NHS Plan. http://www.dh.gov.uk/ prod_consum_dh/groups/dh_digitalassets/@dh/@en/@ps/documents/ digitalasset/dh_118522.pdf. Accessed 1 July 2000.

2. UK Department of Health. Response to reforming NHS financial flows: introducing payment by results. http://www.dh.gov.uk/prod_consum_dh/ groups/dh_digitalassets/@dh/@en/documents/digitalasset/dh_4018704.pdf. Accessed October 2002.

3. Angelillo IF, Ricciardi G, Nante N, Boccia A, Bianco A, La Torre G, et al. Appropriateness of hospital utilisation in Italy. Public Health. 2000;114:9-14.

4. Alijani A, Hanna GB, Ziyaie D, Burns SL, Campbell KL, McMurdo ME, et al. Instrument for objective assessment of appropriateness of surgical bed occupancy: validation study. BMJ. 2003;326:1243-4.

5. Bennett-Guerrero E, Welsby I, Dunn TJ, Young LR, Wahl TA, Diers TL, et al. The use of a postoperative morbidity survey to evaluate patients with prolonged hospitalization after routine, moderate-risk, elective surgery. Anesth Analg. 1999;89:514-9.

6. Grocott MPW, Browne JP, Van der Meulen J, Matejowsky C, Mutch M, Hamilton MA, et al. The Post-Operative Morbidity Survey was validated and used to describe morbidity following major surgery. J Clin Epidemiol. 2007;60:919-28.

7. Bennett-Guerrero E, Feierman DE, Barclay GR, Parides MK, Sheiner PA, Mythen MG, et al. Preoperative and intraoperative predictors of postoperative morbidity, poor graft function, and early rejection in 190 patients undergoing liver transplantation. Arch Surg. 2001;136:1177-83.

8. Wakeling HG, McFall MR, Jenkins CS, Woods WG, Miles WF, Barclay GR, et al. Intraoperative oesophageal Doppler guided fluid management shortens postoperative hospital stay after major bowel surgery. Br J Anaesth. 2005;95:634-42.

9. Webb A. Solutions for reducing length of stay. Health Service Journal. http://www.hsj.co.uk/resource-centre/solutions-for-reducing-length-of-stay/ 373020.article. Accessed 14 January 2008.

10. Gertman PM, Restuccia JD. The appropriateness evaluation protocol: a technique for assessing unnecessary days of hospital care. Med Care. 1981;19:855-71.

11. Kalant N, Berlinquet M, Diodati JG, Dragatakis L, Marcotte F. How valid are utilization review tools in assessing appropriate use of acute care beds? Can Med Assoc J. 2000;162:1809-13.

12. Bentes M, Gonsalves ML, Santos M, Pina E. Design and development of a utilization review program in Portugal. Int J Qual Health Care. 1995;7:201-12.

13. Sangha O, Schneeweiss S, Wildner M, Cook EF, Brennan TA, Witte J, et al. Metric properties of the appropriateness evaluation protocol and predictors of inappropriate hospital use in Germany: an approach using longitudinal patient data. Int J Qual Health Care. 2002;14:483-92.

14. Santos-Eggimann B, Paccaud F, Blanc T. Medical appropriateness of hospital utilization: an overview of the Swiss experience. Int J Qual Health Care. 1995; 7:227-32.

15. D'Alche-Gautier M, Maiza D, Chastang F. Assessing the appropriateness of hospitalisation days in a French university hospital. Int I of Health Care Qual Assurance. 2004;17:87-91.

\section{Submit your next manuscript to BioMed Central and take full advantage of:}

- Convenient online submission

- Thorough peer review

- No space constraints or color figure charges

- Immediate publication on acceptance

- Inclusion in PubMed, CAS, Scopus and Google Scholar

- Research which is freely available for redistribution 